\title{
PROGRAMA DE AVALIAÇÃO DE FORNECEDORES: DESENVOLVIMENTO EM UMA EMPRESA DO SETOR SIDERÚRGICO
}

\section{SUPPLIER EVALUATION PROGRAM: DEVELOPMENT IN A COMPANY FROM THE STEEL SECTOR}

\author{
Vinícius Bittencourt de Almeida*E-mail: v bittencourt@hotmail.com \\ Carla Simone Ruppenthal Neumann* E-mail: csrneumann@ea.ufrgs.br \\ * Universidade Federal do Rio Grande do Sul (UFRGS), Porto Alegre, RS
}

\begin{abstract}
Resumo: Este artigo detalha como foi desenvolvido um programa de avaliação de fornecedores de uma empresa do setor siderúrgico. Para tal, desenvolveu-se um Índice de Qualificação de Fornecedor (IQF), calculado mensalmente através da avaliação dos processos dos fornecedores, dos indicadores de qualidade dos materiais fornecidos e da pontualidade de entregas de materiais. As etapas de desenvolvimento e os resultados obtidos são apresentados para um conjunto de 12 fornecedores em um ciclo completo do programa. Observou-se que a implantação do programa de avaliação motivou uma evolução no IQF dos fornecedores avaliados e mostrou potencial para a expansão aos demais membros da cadeia de suprimentos da empresa.
\end{abstract}

Palavras-chave: Avaliação de desempenho. Fornecedores. Cadeia de suprimentos.

Abstract: This paper details how was developed a supplier evaluation program of a steel industry company. The program develops a Supplier Rating Index (IQF) that is calculated monthly by evaluation of suppliers processes, materials quality provided and timely delivery of materials. The development stages and the results are presented for a set of 12 suppliers in a complete program cycle. It was observed that the implementation of the evaluation program led to an improvement of suppliers' IQF and showed potential for expansion to other members of the company's supply chain.

Keywords: Performance evaluation. Suppliers. Supply chain.

\section{INTRODUÇÃO}

No cenário competitivo atual, podem-se obter vantagens ao estabelecer relações estratégicas com os fornecedores (TUKEL E WASTI, 2001; BIEHL, 2001). Um consumidor não compra um produto de uma empresa, quando o faz, está na verdade comprando de uma cadeia. Isto porque nenhuma empresa compete sozinha em seu segmento, ela precisa que seus fornecedores e seus clientes sejam competitivos para que ela também seja (CAMPOS, 1992; FU E PIPLANI, 2004). 
O sucesso de um negócio depende da habilidade de identificar oportunidades de melhorias e de gerenciar as relações entre os diversos membros que compõe a cadeia de suprimentos (LAMBERT, 2000). Gerenciar esta relação, ou qualquer outro processo, significa monitorar e comparar os resultados com as metas estabelecidas, corrigindo os desvios quando necessário (CAMPOS, 1998). O sucesso de alianças estratégica com os fornecedores depende grandemente do fluxo de informações técnicas trocadas entre os membros da cadeia de suprimentos (MOHR E SPEKMAN, 1994; STUART E McCUTCHEON, 1996; KRAUSE, 1999; GRIFFITHS E MARGETTS, 2000; MILGATE, 2001).

Além do alto custo direto que pode ser causado pelo mau gerenciamento da relação com fornecedores, Taylor e Brunt (2001) destacam que a perda por atraso de entregas do fornecedor e por aquisição de materiais defeituosos são os problemas de maior impacto enfrentados para se atingir a eficiência na cadeia de suprimentos. É prioritário que as empresas trabalhem com seus fornecedores para melhorem continuamente seus índices de desempenho. Observa-se que a parceria com os fornecedores melhora a qualidade e a velocidade de entrega de materiais, permite alterações de projeto mais rápidas, podendo facilitar o desenvolvimento de novos produtos (CRAWFORD E COX,1991).

Pesquisas apontam que fornecedores de classe mundial têm índices de atendimento de pedido entre $90 \%$ e $94 \%$, tempos médios de entrega entre sete e oito dias e índices de erros nos pedidos despachados inferiores a um a cada mil. Isto mostra que custos relacionados ao desabastecimento e não conformidades são preços altos a pagar em um mercado que exige um tempo cada vez menor para entrega de produtos (BALLOU, 2001).

Considerando este cenário, o presente artigo apresenta uma pesquisa ação sobre o programa de avaliação de fornecedores de uma empresa do setor siderúrgico e seus principais fornecedores. O objetivo é apresentar um método de monitoramento e avaliação das práticas realizadas pelos fornecedores e seus respectivos desempenhos no atendimento aos pedidos. Busca-se, no curto prazo, motivar os participantes do programa a monitorar os seus índices. No longo prazo, espera-se elevar os patamares dos índices, gerando a melhoria contínua, o que colabora para aumentar as vantagens da cadeia como um todo. 
Antes de implementar o programa de avaliação, os fornecedores eram monitorados por índices isolados de pontualidade e qualidade no fornecimento, sem um acompanhamento sistemático do setor de suprimentos da empresa siderúrgica. Além disso, alguns índices não se encontravam estabilizados, acarretando o desabastecimento dos setores produtivos, custos com fretes urgentes, aumento de estoques, retrabalho com devoluções de peças e problemas de qualidade no produto final, o que motivou o desenvolvimento do programa de avaliação.

Cabe ressaltar que a empresa estudada já conduzia junto ao Serviço Brasileiro de Apoio ás Micro e Pequenas Empresas (SEBRAE) um programa de desenvolvimento de fornecedores. No entanto, este programa atuava em nível estratégico, auxiliando os fornecedores na elaboração de planejamento estratégico, prospecção de novos mercados, redução do grau de dependência econômica e treinamento em conceitos gerais de qualidade. Entretanto a empresa sentia a necessidade de mensurar o desempenho dos fornecedores segundo critérios quantitativos embasados nas normas e também almejava comparar fornecedores com o mesmo risco de fornecimento.

$\mathrm{O}$ artigo está dividido em cinco seções. Além da presente introdução, o artigo traz na segunda seção uma revisão sobre as normas ISO 9001 e ISO/TS 16949, que foram uma das motivações para desenvolver o programa de avaliação. Na terceira seção é apresentada a revisão sobre avaliação de fornecedores. A quarta seção apresenta o estudo de caso com as etapas utilizadas para a implantação do programa de avaliação e os resultados obtidos. Na seção cinco são apresentadas as conclusões e considerações finais sobre o estudo.

\section{ISO 9001 E ISO/TS 16949}

A ISO 9001 e a ISO/TS 16949 são normas que especificam os requisitos do Sistema de Gestão da Qualidade (SGQ). Um SGQ é um conjunto de regras mínimas que orientam a cada parte da empresa a executar de maneira correta sua função. Ele contribui para assegurar que cada indivíduo não faça seu trabalho da sua maneira, mas que os processos possuam organização e ordem dentro da empresa, garantindo o emprego de recursos de maneira eficiente (MELLO et al, 2009). 
A ISO 9001 teve sua primeira versão em 1987, a partir de um comitê de Garantia da Qualidade do International Organization for Standardization (ISO) e uma nova versão em 2000. A versão de 2000 enfatizou a utilização de uma estrutura relacionada a processos, utilização do sistema de informações para facilitar a melhoria da qualidade e inclusão da satisfação do cliente para melhoria das atividades (KARTHA, 2003). Sua última revisão foi realizada no final de 2008 , sendo que essa revisão contemplou aperfeiçoamentos na clareza e na tradução, verificou a sua compatibilidade com a ISO 14000, mantendo-se inalterada nas questões essenciais da versão de 2000. De acordo com Haro (2001), devido ao alto nível no ramo automobilístico a norma ISO 9001 tornou-se insuficiente, pois era muito genérica e não abordava as exigências do setor. Surgiu assim a ISO/TS 16949 que eliminou a necessidade de certificações múltiplas para atender a diferentes países/setores (KARTHA, 2003).

A ISO/TS 16949 teve sua primeira versão em 1999, sendo elaborada pelo International Automotive Task Force (IATF) que é composto por um grupo de oito fabricantes de automóveis e tem na sua essência a ISO 9001, com inclusão de ferramentas de controle e adaptações para o setor automotivo. Essa norma especifica os requisitos do sistema da qualidade para projeto/desenvolvimento, produção, instalação e assistência técnica de produtos relacionados à indústria automotiva. Ao compreender a interação dos processos permite uma possível melhoria do produto e da qualidade do processo, e evita variações na cadeia de fornecimento (KARTHA, 2003). Sua última atualização foi em 2010 e constitui requisito básico para uma empresa fornecer para o setor automotivo.

Essas duas normas possuem uma abordagem de processo em sua essência e utilizam o PDCA (sigla provinda do inglês Plan, Do, Check e Act) como ferramenta para execução do processo de melhoria. Estabelecem requisitos e padrões genéricos que podem ser utilizados para avaliar a capacidade de uma organização em atender as especificações do cliente. Alguns dos requisitos abordados pelas normas ISO 9001 e ISO/TS 16949 dizem respeito a: responsabilidade da direção, política da qualidade, planejamento da realização do produto, aquisição, controle de equipamentos de medição, controle de não conformidades, medição e análise e melhoria (ABNT, 2008). Mais especificamente no processo de aquisição, a empresa compradora deve assegurar que o produto esteja de acordo com o que foi 
especificado e cabe a ela estabelecer critérios para seleção, avaliação e reavaliação. O fornecedor deve ser avaliado quanto à conformidade dos requisitos do produto entregue, interrupções no cliente, desempenho na programação de entrega e notificações sobre situações especiais relacionadas às questões de qualidade ou entrega. Por fim, a organização deve incentivar o fornecedor a monitorar o desempenho de seus processos de manufatura (ABNT, 2010).

\section{AVALIAÇÃO DE FORNECEDORES}

Para este trabalho, procurou-se na literatura diferentes abordagens de relacionamento com fornecedores, ressaltando a importância de uma relação em que tanto a empresa compradora quanto o fornecedor usufrua dos benefícios da melhoria do desempenho. As abordagens de relacionamento com fornecedores podem ser resumidas em duas grandes linhas de pensamento. A primeira é chamada de competitiva, na qual o objetivo principal é a redução de custos, é caracterizada pela disputa entre adversários, baseada em fornecedores múltiplos, oferta competitiva e utilização de tempos de contrato curtos. A segunda é chamada de abordagem de parceria colaborativa, a qual requer acordos de longo prazo focando na qualidade dos produtos e do atendimento, além do custo. A abordagem de parceria colaborativa requer compartilhamento de informações, melhoria contínua de ambas as partes e geralmente é estabelecida por iniciativa da empresa compradora que precisa garantir que seus fornecedores sejam competitivos. Um dos recursos utilizados para garantir a competitividade e o alto desempenho dos fornecedores são os programas de avaliações de fornecedores realizados pela própria empresa compradora (LAMMING, 1993; WATTS E HAHN, 1993; CARTER et al., 1998 e HUMPHREYS et al.,2001).

A necessidade de uma avaliação estruturada é reconhecida pelas principais empresas compradoras do mundo desde o início dos anos noventa (STUELAND, 2004). A avaliação do fornecedor estabelece os critérios para fornecimento, garantindo à empresa compradora a segurança necessária para a aquisição de materiais ou serviços sem riscos para seu negócio. A avaliação de fornecedor deve ser encarada como uma forma de estreitamento de relações entre as empresas, 
promovendo um ambiente de melhoria contínua em uma relação ganha-ganha (BAILY, 2000).

A avaliação de fornecedores pode ser parte de um programa de desenvolvimento de fornecedores, o qual é caracterizado pelo investimento de recursos da empresa compradora nos processos dos fornecedores como exemplificado por Neumann (2004), através de um estudo de caso de implantação de um sistema de troca rápida de ferramentas em fornecedor de uma empresa do setor de máquinas agrícolas. A avaliação também pode ser conduzida isoladamente, apenas como um critério de seleção ou monitoramento de fornecedores, como exemplificado por Rosa et al. (2006) em seu estudo de caso de avaliação multicriterial de fornecedores, por Rosa e Vaccaro(2009) ao monitorar as perdas dos processos, por Seidel et al. (2011) utilizando o controle estatístico de processos e por Estorilio e Posso (2011) ao analisar o modo e efeito das falhas de um conjunto de fornecedores.

Tracey e Tan (2001) afirmam que os indicadores de satisfação do cliente final, como preço competitivo, qualidade dos produtos e alto nível de serviço nas entregas dos produtos são diretamente afetados pelos fornecedores de uma empresa. O mesmo autor ressalta a importância do envolvimento de fornecedores em programas de desenvolvimento e avaliações para a melhoria contínua de seus processos. Krause (1997) apresenta os impactos do desenvolvimento e avaliação de fornecedores nos indicadores de qualidade, confiabilidade e tempo de entrega dos mesmos. As ações implementadas pelas empresas respondentes implicaram em uma redução média de $6,2 \%$ no número de materiais entregues com defeito pelos seus fornecedores. O percentual de entregas no prazo apresentou em média um aumento de $11,17 \%$, enquanto houve uma redução média de 14 dias no tempo total de entrega de materiais após as ações junto aos fornecedores.

Não existe consenso entre quais requisitos devem ser avaliados nos fornecedores. Mas assim como os indicadores de desempenho de uma empresa devem sustentar a estratégia da corporação, os indicadores de desempenho para avaliação dos fornecedores também auxiliam a empresa cliente a alcançar seus objetivos. A avaliação do fornecedor, portanto, deve estar alinhada com o planejamento estratégico da organização (MERLI, 1998). Rosa et al (2006) apresentam uma avaliação de fornecedores baseada em uma análise multicriterial, 
nas quais os fatores avaliados e seus pesos foram: (i) qualidade $(30,8 \%)$, (ii) entrega $(24,3 \%)$, (iii) preço $(28,7 \%)$, (iv) flexibilidade $(7,1 \%)$ e $(v)$ tecnologia $(9,1 \%)$. Tanto a definição das importâncias relativas de cada fator como a avaliação de cada fornecedor foi realizada subjetivamente por especialistas do setor de suprimentos da empresa estudada, utilizando a análise hierárquica de processos. Stueland (2004) conclui que os critérios de avaliação mais utilizados por diversas empresas foram os de qualidade, nível de serviço de entregas e custos totais, sendo que os critérios de qualidade em média recebem relevância maior nas avaliações. O autor também ressalta a importância do respaldo da alta direção de uma empresa no processo de avaliação de fornecedores, destacando como fator fundamental para o sucesso desses tipos de ações.

Para Merli (1998) os critérios de avaliação de fornecedores devem variar de acordo com o nível de relacionamento que a empresa possui com o fornecedor. Fornecedores comuns, por exemplo, devem ser avaliados em termos de custo, qualidade dos produtos, tempos de entrega e confiabilidade de entrega. Conforme o crescimento da relação entre as empresas, fatores como, sistemas de garantia da qualidade, nível tecnológico, flexibilidade e capacidade do negócio devem ser incorporados nas avaliações.

Por fim, pode restar a dúvida de como escolher os participantes de um programa de avaliação. De acordo com Kraljic (1983) os participantes de um programa podem ser escolhidos utilizando uma matriz que classifica os materiais de acordo com o seu risco de fornecimento, levando em consideração o número de fontes qualificadas disponíveis, e também uma análise da importância do material para a operação e resultado da empresa, conforme apresentado na Figura 1.

No primeiro quadrante da Figura 1 estão aqueles materiais com muitas fontes de fornecimento disponíveis no mercado e com relevância baixa. Geralmente, para estes materiais, a relação com fornecedores se estabelece através de acordos corporativos, que visam ganhos de escala, visto que o valor unitário dos itens não é alto. No segundo quadrante estão itens com muitas fontes de fornecimento, mas cujos itens têm muita relevância no resultado da empresa. A prática de mercado para estes itens é estimular a competitividade entre os fornecedores, buscando fontes alternativas e redução de custo. Os itens do terceiro quadrante são considerados estratégicos e as práticas de mercado para estes são acordos de 
longo prazo com os fornecedores, negociados pela alta direção da empresa, devido ao grande poder de barganha do fornecedor. Os itens do quarto quadrante são considerados itens de risco devido à escassez de fontes de suprimento. Como prática de mercado para esses itens é comum buscar a padronização e a simplificação de componentes, levando os materiais para o primeiro quadrante, fugindo de fornecedores exclusivos (Kraljic, 1983). Cabe à empresa compradora verificar sua estratégia e definir um grupo de fornecedores de determinado quadrante para participar do programa de avaliação.

Figura 1 - Matriz de Kraljic

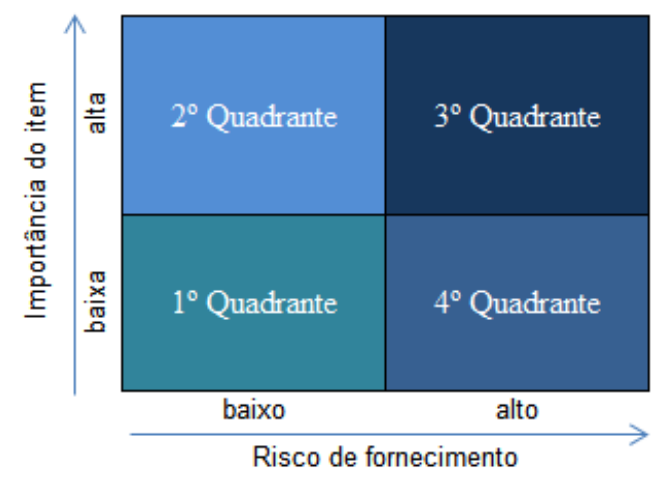

\section{PROCEDIMENTOS METODOLÓGICOS E APLICAÇÃO DO PROGRAMA DE AVALIAÇÃO DE FORNECEDORES}

O programa de avaliação de fornecedores foi desenvolvido por uma empresa do setor siderúrgico. Este programa surgiu a partir de um esforço conjunto dos setores de gestão da qualidade, suprimentos e administração de materiais da empresa. Complementarmente, o programa permite o atendimento aos requisitos da ISO 9001 e ISO/TS 16949 relativos à gestão da cadeia de fornecimento. O seu sistema de avaliação cíclico, com fornecimento de feedback e comprovação de melhorias nos fornecedores auxilia na redução de interrupção de produção por problemas de qualidade ou atrasos do fornecedor, na redução de estoques, tornando a cadeia mais confiável e competitiva. 


\subsection{Método utilizado}

Sob o ponto-de-vista da natureza, esta pesquisa é classificada como aplicada, visto que gera conhecimentos úteis à solução de problemas reais; sob o ponto-devista da forma de abordagem do problema, esta pesquisa é classificada como quantitativa; sob o ponto-de-vista de seus objetivos, é classificada como explicativa, pois visa identificar os fatores que contribuem para avaliar os fornecedores e aprofunda o conhecimento da realidade; sob o ponto-de-vista dos procedimentos técnicos é classificada como pesquisa-ação, pois foi concebida e realizada para resolver o problema coletivo da empresa, estando os pesquisadores envolvidos na resolução dos problemas de modo colaborativo Após a escolha do tema, buscou-se na literatura compreender sob quais aspectos os fornecedores podem ser avaliados e então se iniciou a construção do programa de avaliação (GIL, 2010; SILVA E MENEZES, 2005).

O programa de avaliação de fornecedores estabelece um Índice de Qualificação de Fornecedor (IQF) atualizado mensalmente, composto por três indicadores ponderados de acordo com a necessidade da empresa compradora. Os indicadores monitorados são: (i) a avaliação formal dos processos do fornecedor, (ii) o indicador de qualidade de produtos entregues e (iii) o indicador de pontualidade nas entregas de materiais (ALMEIDA, 2011).

O programa é dividido em oito etapas distribuídas dentro de um ciclo PDCA (Figura 2). A fase de Planejamento prevê as etapas de (i) elaboração do checklist de avaliação do fornecedor e (ii) definição dos fornecedores a serem avaliados. A fase de Execução compreende as etapas de (iii) aplicação do checklist nos fornecedores, (iv) cálculo do IQF e (v) feedback ao fornecedor. A fase de Verificação é composta pelas etapas de (vi) revisão do checklist e (vii) reaplicação do checklist e atualização do IQF. A fase de Atuação compreende (viii) a verificação dos resultados do programa de avaliação. A Figura 2 também ilustra a etapa de elaboração do plano de ação que é de responsabilidade do fornecedor e não é descrita neste trabalho. 
Figura 2 - Programa de avaliação de fornecedores

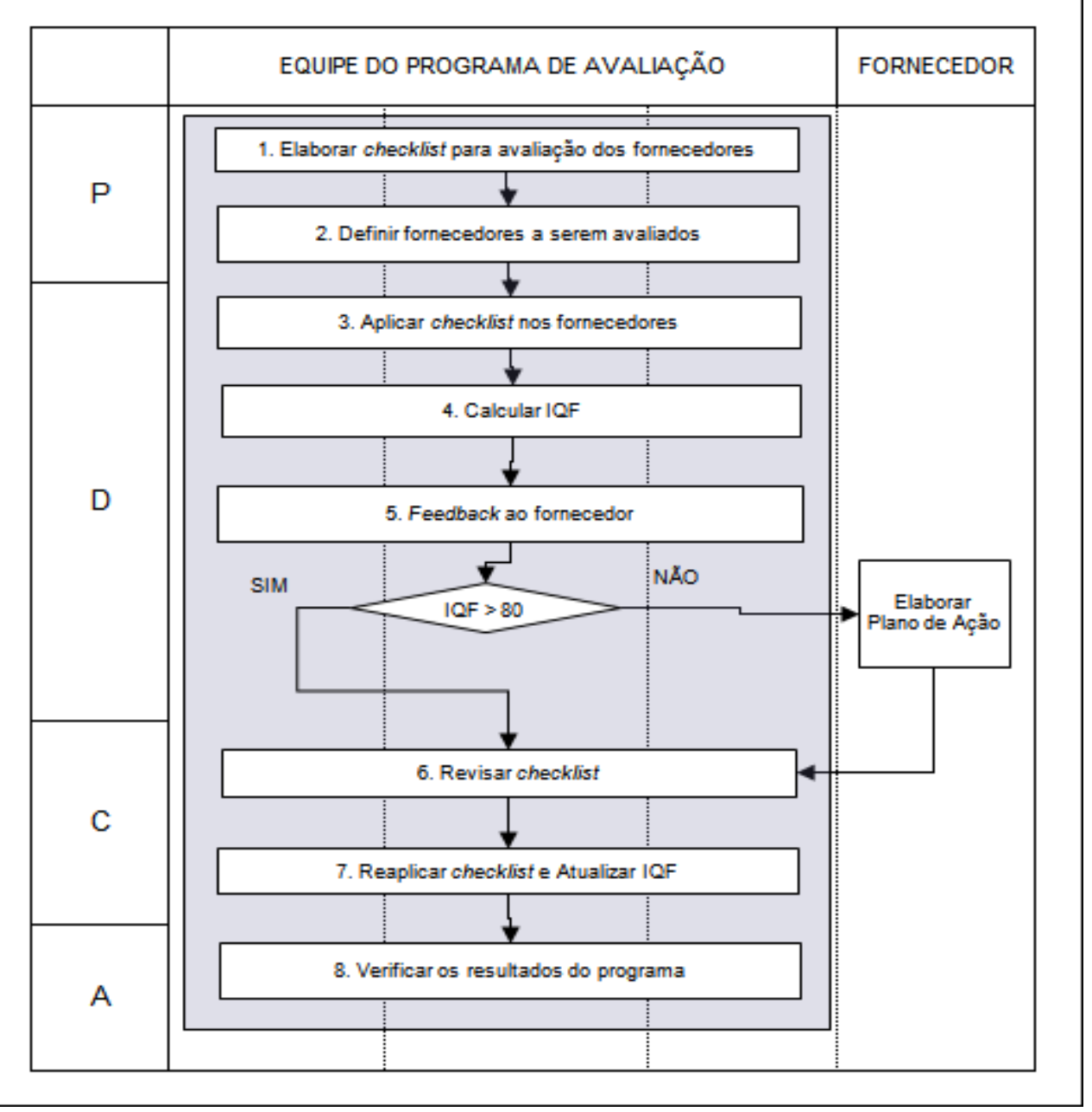

A primeira etapa consiste na criação de um checklist que possa evidenciar as melhores práticas do fornecedor. Através de uma reunião da equipe formada no início do programa é realizado um brainstorm apoiado nas normas internacionais de qualidade, como por exemplo a ISO 9001 e ISO/TS 16949 para levantamento de aspectos importantes para empresa compradora que devem ser encontrados nos fornecedores. Nessas normas, verifica-se que um SGQ é avaliado através do grau de atendimento a alguns requisitos como, por exemplo, política de qualidade, controle de não conformidades, medição, análise e melhoria, planejamento da realização do produto e responsabilidade da direção. Cabe à empresa que deseja avaliar seus fornecedores adaptar estes requisitos para a sua realidade, criando um checklist que atenda suas necessidades, pois a norma não especifica a forma de avaliação dos fornecedores. 
Cada requisito de avaliação é composto por um conjunto de questões que irão evidenciar as práticas adotadas pelo fornecedor. O nível de atendimento de cada questão do checklist pode variar de 0 (zero) a 4 (quatro) pontos de acordo com as evidências encontradas e consenso entre a equipe avaliadora e o fornecedor. $A$ nota 4 representa o conceito excelente e o pleno atendimento ao requisito. A nota 3 é um conceito bom e representa o atendimento básico ao requisito. A nota 2 (conceito regular) identifica que são necessárias melhorias. A nota 1 representa um conceito de iniciante no processo avaliado, mas o fornecedor não atende ao requisito solicitado. A nota 0 representa um conceito ruim, no qual não são identificadas evidências do requisito avaliado. Também existe a possibilidade do requisito não ser aplicável na avaliação, para o caso de questões identificadas como não relevantes para determinado fornecedor. A nota final do questionário consiste na soma total dos pontos obtidos dividida pela soma da pontuação máxima das questões aplicáveis.

Na segunda etapa é realizada a definição dos fornecedores para participação no programa de avaliação. Esta etapa inicia com a classificação dos fornecedores de acordo com o tipo de material entregue, analisados por especialistas do setor de suprimentos. Os fornecedores escolhidos para serem avaliados são comunicados pelo setor de suprimentos e sua participação é opcional.

A terceira etapa é a aplicação do checklist no fornecedor que deve ser executada nas dependências do mesmo, tendo a duração estimada de três horas. Essa avaliação é executada por integrantes da equipe formada no início do programa, que também constitui a equipe avaliadora. A pontuação do checklist deve ser preenchida através de um consenso entre a equipe avaliadora e os representantes do fornecedor, sendo que este último deve apresentar evidências do atendimento aos requisitos.

$\mathrm{Na}$ quarta etapa, após a pontuação da avaliação dos processos do fornecedor, é possível apurar os resultados mensais do $\mathrm{IQF}_{n, m}$ em que o índice $n$ indica o ano e o índice $m$ indica o mês de avaliação dentro do programa. O IQF ${ }_{n, m}$ consiste em uma soma ponderada da avaliação através do checklist $\left(C L_{n}\right)$, da pontuação referente a não conformidades $\left(\mathrm{NC}_{n, m}\right)$ verificadas no recebimento de materiais e da pontualidade de entregas do fornecedor $\left(P E_{n, m}\right)$, conforme a equação (1). O peso atribuído ao índice $\mathrm{CL}_{n, m}$ na equação (1), revela a grande importância 
dada a avaliação através do checklist, pois acredita-se que a execução de boas práticas nos processos da empresa acarretam em resultados positivos nos demais indicadores.

$$
\mathrm{IQF} n, m=0,5 \times \mathrm{CL} n+0,4 \times \mathrm{NC} n, m+0,1 \times \mathrm{PE} n, m
$$

A pontuação $\mathrm{NC}_{n, m}$ é dada através da razão entre o número de não conformidades detectadas nos últimos doze meses e o número de itens entregues neste mesmo período, seguindo a pontuação mostrada no Quadro 1. Na empresa do estudo, esta pontuação foi obtida através da análise do histórico de desempenho dos fornecedores da empresa estudada, no qual os fornecedores de melhor desempenho apresentaram $0 \%$ de não conformidades, enquanto os de pior desempenho apresentaram um percentual acima de $8 \%$. Salienta-se que podem ser necessárias algumas adaptações nesses percentuais em outras aplicações.

Quadro 1 - Pontuação de não conformidades $\left(\mathrm{NC}_{n, m}\right)$

\begin{tabular}{cc}
\hline Percentual Não Conformidade (\%) & ${\text { Pontuação } \mathbf{N C}_{\boldsymbol{n}, \boldsymbol{m}}}^{\text {Pan }}$ \\
\hline 0 a 2 & 100 \\
2 a 4 & 80 \\
4 a 6 & 60 \\
6 a 8 & 40 \\
acima de 8 & 20 \\
\hline
\end{tabular}

A pontuação $\mathrm{PE}_{n, m}$ é dada através do número de itens entregues dentro do prazo acordado, dividido pelo número total de itens entregues nos últimos doze meses e multiplicado por cem. Esta forma revela-se simples e eficaz, pois reflete o percentual de atendimento aos prazos de entrega que foram previamente acordados entre o contratante e contratado.

Ao longo do ano, o IQF ${ }_{n, m}$ é recalculado mensalmente através da atualização dos índices $\mathrm{NC}_{n, m}$ e $\mathrm{PE}_{n, m}$ e da manutenção do valor obtido na avaliação anual do $\mathrm{CL}_{n}$. Assim a parcela do $\mathrm{CL}_{n}$ será a mesma nos doze meses seguintes, até que seja feita nova avaliação $\mathrm{CL}_{n+1}$.

Pela experiência dos membros da empresa, uma vez atingidos 80 pontos no $\mathrm{IQF}_{n, m}$ em qualquer mês de medição, o fornecedor é considerado homologado pela empresa compradora e este status permanece até a próxima avaliação anual para 
apuração do índice $\mathrm{CL}_{n}$. Se no mês seguinte a atualização do $\mathrm{CL}_{n}$ o fornecedor mantiver o $\mathrm{IQF}_{n, m}$ em 80 pontos ou mais, ele permanece homologado até a próxima atualização do $\mathrm{CL}_{n}$, do contrário ele será considerado não homologado até que alguma medição mensal do $\mathrm{IQF}_{n, m}$ atinja o mínimo de 80 pontos.

A quinta etapa do procedimento fornece um feedback da avaliação ao fornecedor. Esse feedback é realizado em uma reunião entre a equipe avaliadora e a direção da empresa fornecedora e ocorre sempre no mês seguinte a atualização do índice $C L_{n}$. Nesta reunião é apresentado o IQF do fornecedor, assim como o desempenho do fornecedor em cada índice que compõe o IQF. Discutem-se principalmente os pontos negativos que devem ser corrigidos e melhorados decorrentes da avaliação do checklist e os pontos positivos que devem ser mantidos.

A reunião de feedback busca comprometer a direção da empresa fornecedora com o programa de avaliação para que efetuem as correções necessárias e sejam verificadas melhorias efetivas nos indicadores de desempenho. Decorridos trinta dias após o feedback, as empresas com status de não homologadas no mês seguinte a execução da avaliação para atualização do índice $\mathrm{CL}_{n}$ devem apresentar um plano de ação para ser executado ao longo de um ano. O plano deve focar na melhoria dos requisitos do checklist e também na melhoria dos indicadores de desempenho.

A sexta etapa consiste em uma reunião da equipe do projeto para a revisão do checklist de avaliação dos fornecedores. Através de um brainstorm baseado em possíveis atualizações das normas ISO 9001 e ISO/TS 16949 e em uma análise da estratégia e objetivos da empresa, que podem variar de um ano para o outro, são propostas inclusões ou exclusões de novas questões para o checklist.

A sétima etapa consiste em uma reaplicação do checklist revisado e atualização do $\mathrm{IQF}_{n, m}$. Assim como na terceira etapa, a equipe avaliadora reavalia a pontuação de cada questão do checklist decorrido um ano da avaliação anterior. $I_{Q} F_{n, m}$ dos fornecedores sofrerá uma atualização nas três parcelas após a reaplicação do checklist revisado nos fornecedores.

A oitava etapa consiste na avaliação dos resultados do programa. Nesta etapa os representantes da equipe do programa verificam a evolução do $I_{n, m}$ mensal de cada fornecedor, assim como o desempenho de cada índice 
individualmente, através da análise estatística das pontuações. Espera-se que uma boa avaliação $\mathrm{CL}_{n}$, que representa a avaliação dos processos e dos meios de produção, reflita em um bom desempenho final nos índices $\mathrm{NC}_{n, m}$ e $\mathrm{PE}_{n, m}$. Nesta etapa também definem-se ações para aqueles fornecedores que não atingiram a pontuação mínima para homologação, como o descredenciamento para fornecimento ou permanência no programa de avaliação por mais um ano.

A oitava etapa encerra o período de um ano do programa, e após sua execução, conforme ilustra a Figura 1, o programa retorna para quinta etapa, que consiste em fornecer feedback ao fornecedor, assumindo um caráter cíclico. Esperase com esse programa construir na cadeia de fornecedores um compromisso de melhoria contínua nos processos e nos indicadores monitorados, trazendo benefícios tanto para a empresa compradora, que receberá um nível de serviço melhor, quanto para o negócio de seus fornecedores, que se mantêm competitivos no mercado.

\subsection{Resultados do programa de avaliação de fornecedores}

A empresa em estudo é uma multinacional brasileira do setor siderúrgico. As ações foram executadas na cadeia de fornecedores de uma de suas usinas no sul do Brasil. A empresa, no entanto, está presente em 14 países e atende os mercados de construção civil, agropecuária e indústria em geral, fornecendo há mais de 100 anos aços das mais variadas composições e apresentações como: vergalhões, aços para construção mecânica, telas e pregos. A empresa conserva traços familiares, está na sua quinta geração na sucessão de liderança e tem preocupação com a sustentabilidade econômica, social e ambiental. Como ação de sustentabilidade econômica interage com a comunidade onde atua, procurando, por exemplo, executar ações em conjunto com a cadeia de fornecimento na região em que está inserida, convertendo essas ações em benefícios para o próprio negócio.

O programa de avaliação de fornecedores representa uma dessas ações, no qual uma ação conjunta dos setores de gestão da qualidade, suprimentos e administração de materiais buscou trazer resultados positivos para todas as empresas envolvidas. Cinco profissionais representando os setores citados 
anteriormente formaram a equipe do projeto e foram responsáveis pela execução do estudo de caso descrito a seguir.

A primeira etapa consistiu na criação do checklist para avaliação dos fornecedores. Em uma reunião de aproximadamente quatro horas, baseando-se em documentos de avaliação para obtenção da certificação da ISO 9001 e da ISO/TS16949, a equipe do projeto chegou a cinco requisitos fundamentais que deveriam ser avaliados nos fornecedores: (i) análise crítica de pedido de compra ou contrato de fornecimento, (ii) aquisição de matéria prima e insumos, (iii) organização da produção, (iv) qualidade e (v) expedição de produtos. A Figura 3 apresenta o checklist criado com o conjunto de questões elaboradas para os cinco requisitos propostos, assim como a escala de pontuação que pode ser atingida pelo fornecedor.

Figura 3 - Checklist de avaliação em fornecedor

\begin{tabular}{|c|c|c|c|c|c|c|}
\hline \multirow{2}{*}{ RT. ANLLISE CPIIKCA DO PEDIDO DE COMPRU CU CONTRATO DE FORNECCMENTO } & \multicolumn{6}{|c|}{$\rightarrow$} \\
\hline & 0 & 1 & 2 & 3 & 4 & NA \\
\hline \multicolumn{7}{|l|}{ A Empresa realza para todas as solotaçōes de cotaçăo um orçamento e este ê castredver? } \\
\hline \multicolumn{7}{|l|}{ 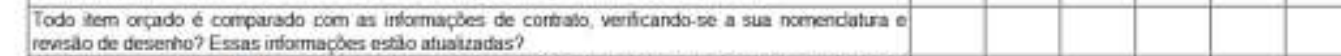 } \\
\hline \multicolumn{7}{|l|}{ 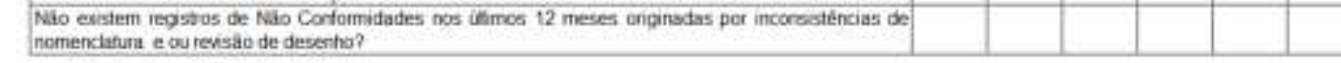 } \\
\hline \multicolumn{7}{|l|}{ 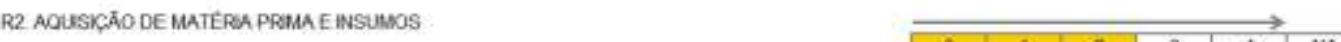 } \\
\hline & 0 & 1 & 2 & 3 & 4 & MA \\
\hline \multicolumn{7}{|l|}{ 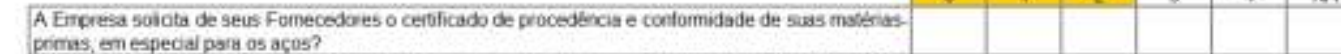 } \\
\hline \multicolumn{7}{|l|}{ 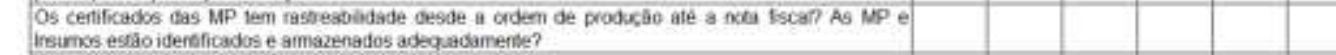 } \\
\hline \multicolumn{7}{|l|}{ 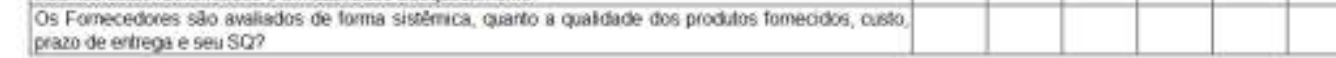 } \\
\hline \multirow{2}{*}{ R3 ORGANZACALO DA PROOUCAO } & & & & & & \\
\hline & 0 & 1 & 2 & 3 & 4 & $\mathrm{MA}$ \\
\hline \multicolumn{7}{|l|}{ 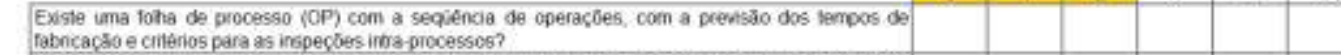 } \\
\hline \multicolumn{7}{|l|}{ 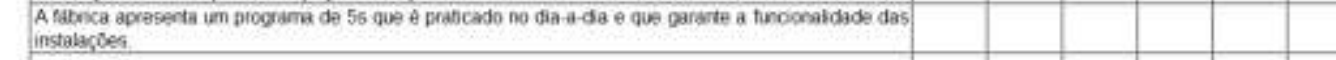 } \\
\hline \multicolumn{7}{|l|}{ 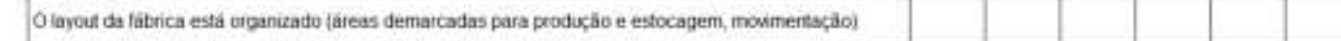 } \\
\hline \multirow[t]{2}{*}{ RA OUAUDADE } & & & & & & \\
\hline & 0 & 1 & 2 & 3 & 4 & NA \\
\hline \multicolumn{7}{|l|}{ 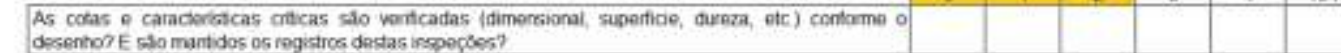 } \\
\hline \multicolumn{7}{|l|}{ 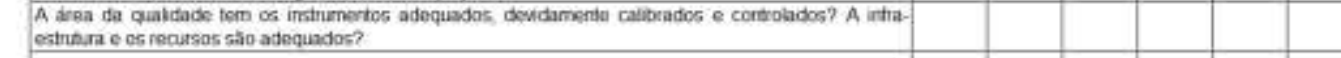 } \\
\hline \multicolumn{7}{|l|}{ A Empresa tem procesmentos ittemos para o geu Sisterna de Gestāo da Quấdade? } \\
\hline \multicolumn{7}{|l|}{ Como voce (Oveça) dassitca o Sistema da Gestăo da Cialidade da swa Empresa? } \\
\hline \multirow[t]{2}{*}{ R5. EXPEDICĀO OE PRODUTOS } & & & & & & \\
\hline & 0 & 1 & 2 & 3 & 4 & NA \\
\hline \multicolumn{7}{|l|}{ 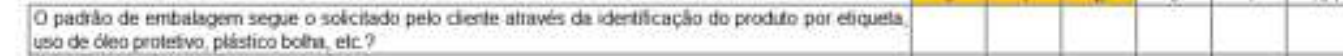 } \\
\hline 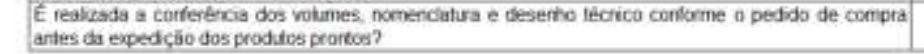 & & & & & & \\
\hline
\end{tabular}

A segunda etapa, que consistiu na definição dos fornecedores a serem avaliados, ocorreu reunindo-se três experts da área de suprimentos da empresa Revista Produção Online, Florianópolis, SC, v.13, n. 4, p. 1569-1595, out./dez. 2013. 
para classificar quarenta e quatro grupos de materiais já existentes quanto ao seu risco de fornecimento e importância para o processo produtivo e resultado da empresa. O processo foi organizado pela equipe de projeto e ocorreu de forma simples devido ao grau de experiência dos profissionais envolvidos na classificação. A Figura 4 exemplifica a matriz de posicionamento de materiais para a empresa estudada.

Os itens do primeiro e quarto quadrante são itens de baixa complexidade e por esse motivo não foram priorizados no programa de avaliação. Os itens do terceiro quadrante são itens cujos fornecedores são lideres em suas cadeias de fornecimento, possuem certificações de qualidade reconhecidas internacionalmente e em sua maioria possuem exclusividade de fornecimento de seus produtos. A equipe decidiu aplicar o programa de avaliação nos fornecedores de itens pertencentes ao segundo quadrante.

Figura 4 - Matriz de Kraljic da empresa estudada

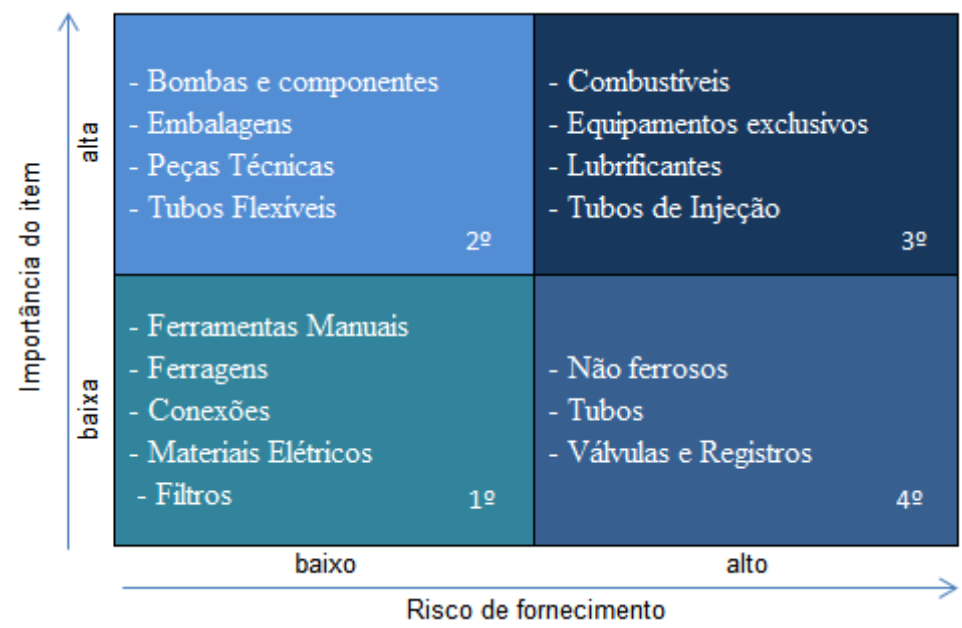

O segundo quadrante possui materiais com alta importância para o processo produtivo, cuja falta ou a qualidade tem grande impacto para o negócio da empresa. Apesar de a literatura recomendar uma estratégia de redução de custo através de substituição de fornecedores, a criticidade do item foi avaliada como determinante para esta escolha, em detrimento ao impacto financeiro das categorias. Assim, para alguns materiais deste quadrante preferiu-se o desenvolvimento e avaliação de fornecedores à substituição dos mesmos. 
Dentro do segundo quadrante, os fornecedores ainda foram priorizados pelo Princípio de Pareto optando pelas relações A de faturamento com a empresa compradora (preço unitário x quantidade) para aplicação do programa de avaliação. O grupo de fornecedores escolhido é constituído de doze empresas, das quais onze enquadram-se no grupo de fornecimento de peças técnicas sob desenho e uma empresa é fornecedora do grupo de tubos flexíveis.

Este grupo de fornecedores se enquadra entre micro e pequenas empresas. As empresas fornecedoras de peças técnicas sob projeto possuem equipamentos de usinagem, como tornos CNC e fresadoras, assim como equipamentos de calderaria, como máquinas de corte e solda. A empresa fornecedora de tubos flexíveis é uma empresa que possui equipamentos de montagem de mangueiras hidráulicas destinadas à passagem de fluídos.

A execução da terceira etapa foi realizada pela equipe do projeto nas dependências do fornecedor conforme procedimento metodológico e teve a duração de aproximadamente dois meses. A Tabela 1 apresenta as médias das pontuações de cada fornecedor nos diferentes requisitos do checklist, assim como a pontuação $\mathrm{CL}_{1}$ apresentada por cada fornecedor.

Tabela 1 - Avaliação do checklist $\left(\mathrm{CL}_{1}\right)$

\begin{tabular}{ccccccc}
\hline Fornecedor & $\mathbf{R} 1$ & $\mathbf{R 2}$ & $\mathbf{R 3}$ & $\mathbf{R 4}$ & $\mathbf{R 5}$ & $\mathbf{C L}_{\mathbf{1}}$ \\
\hline F1 & 3,33 & 2,00 & 2,67 & 1,50 & 4,00 & 63,33 \\
F2 & 4,00 & 2,00 & 2,00 & 1,75 & 3,00 & 61,70 \\
F3 & 2,67 & 1,67 & 1,33 & 1,25 & 3,00 & 46,70 \\
F4 & 3,67 & 3,00 & 3,00 & 3,25 & 4,00 & 80,00 \\
F5 & 3,67 & 2,00 & 1,67 & 2,00 & 3,50 & 61,70 \\
F6 & 3,33 & 2,00 & 1,33 & 3,50 & 4,00 & 70,00 \\
F7 & 3,67 & 3,00 & 2,33 & 3,25 & 3,00 & 76,70 \\
F8 & 2,67 & 2,00 & 1,00 & 1,50 & 3,50 & 50,00 \\
F9 & 4,00 & 2,00 & 3,33 & 1,50 & 3,50 & 68,30 \\
F10 & 3,67 & 4,00 & 2,67 & 3,75 & 4,00 & 90,00 \\
F11 & 4,00 & 2,33 & 2,33 & 2,00 & 3,00 & 63,30 \\
F12 & 4,00 & 1,67 & 3,00 & 2,25 & 4,00 & 71,70 \\
Média & 3,56 & 2,31 & 2,22 & 2,29 & 3,54 & \\
\hline
\end{tabular}

Uma análise revela que os requisitos que apresentaram melhor desempenho foram o R1 e o R5. O R1 apresentou média de 3,56 pontos nos fornecedores avaliados, evidenciando uma boa prática na rastreabilidade de orçamentos, assim como na análise das condições comerciais do pedido, fundamentais para evitar 
divergências posteriores no preço da nota fiscal ou especificação do produto expedido. O R5 apresentou média de 3,54 pontos. Os fornecedores em geral atendem aos requisitos de embalagem e identificação dos produtos, além de realizarem a conferência entre a nota fiscal e a ordem de venda emitida.

Por outro lado, os requisitos R2, R3 e R4 não apresentaram um bom desempenho nos fornecedores. No R2 verificou-se que apesar dos fornecedores apresentarem certificados para suas matérias primas, esses certificados não possuíam rastreabilidade dentro da empresa, não sendo possível identificar, na maioria dos casos, qual lote de matéria prima foi utilizado em determinado produto expedido. Além disto, apenas quatro fornecedores apresentaram evidências de um sistema de avaliação de sua cadeia de fornecimento.

No R3 os fornecedores apresentam fragilidades, não possuindo em média elementos básicos como folha de processo, $5 \mathrm{~S}$ 's e demarcação de áreas produtivas. O R4 também não apresentou bons resultados. Apesar de 100\% dos fornecedores apresentarem algum método de verificação da qualidade, como aferições com instrumentos específicos para determinadas peças, verificou-se em média a falta de um sistema que garantisse a correta calibragem dos instrumentos, impossibilitando uma boa qualidade na medição de cotas críticas, por exemplo.

A quarta etapa do procedimento efetua o cálculo do $\mathrm{IQF}_{n, m}$, conforme equação 1 , de cada fornecedor para cada mês do ano. A Tabela 2 apresenta o resultado do $I Q F_{1,1}$ de cada fornecedor. Verifica-se que os fornecedores $F 1, F 4, F 7$, F10 e F12 obtiveram pontuação para tornarem-se fornecedores homologados, pois atingiram um IQF 1,1 igual ou superior a oitenta pontos.

Tabela 2 - Índice de Qualificação de Fornecedor

\begin{tabular}{ccccc}
\hline Fornecedor & $\mathbf{C L}_{1}$ & $\mathbf{P E}_{1,1}$ & $\mathbf{N C}_{1,1}$ & $\mathbf{I Q F}_{1,1}$ \\
\hline F1 & 63,33 & 83,60 & 100 & 80,03 \\
F2 & 61,70 & 94,53 & 80 & 72,30 \\
F3 & 46,70 & 95,14 & 80 & 64,86 \\
F4 & 80,00 & 86,81 & 80 & 80,68 \\
F5 & 61,70 & 82,69 & 80 & 71,12 \\
F6 & 70,00 & 78,13 & 80 & 74,81 \\
F7 & 76,70 & 98,50 & 100 & 88,20 \\
F8 & 50,00 & 64,74 & 40 & 47,47 \\
F9 & 68,30 & 76,32 & 80 & 73,78 \\
F10 & 90,00 & 87,72 & 80 & 85,77 \\
F11 & 63,30 & 93,78 & 80 & 73,03 \\
F12 & 71,70 & 75,97 & 100 & 83,45 \\
\hline
\end{tabular}

Revista Produção Online, Florianópolis, SC, v.13, n. 4, p. 1569-1595, out./dez. 2013. 
Os resultados foram apresentados aos fornecedores como previsto na quinta etapa. A equipe avaliadora apresentou os resultados obtidos para as direções das empresas, buscando o comprometimento para melhoria tanto da avaliação $\mathrm{CL}_{n}$ quanto dos indicadores $\mathrm{PE}_{n, m}$ e $\mathrm{NC}_{n, m}$. Após o feedback, os fornecedores que no $\mathrm{IQF}_{1,1}$ não apresentaram pontuação suficiente para homologação tiveram o prazo de trinta dias para apresentação do plano de ação.

No feedback aos fornecedores observou-se uma grande receptividade na assimilação de suas limitações e pontos negativos do checklist de avaliação. No entanto essa receptividade percebida na maioria não se confirmou na totalidade do cumprimento da etapa de criação do plano de ação. Dois fornecedores não apresentaram ou não executaram o plano de ação, alegando falta de recursos ou tempo para execução da atividade.

A sexta etapa foi executada pela equipe do projeto e consistiu na revisão do checklist de avaliação de fornecedores. A equipe se reuniu por aproximadamente quatro horas para a verificação das normas internacionais de qualidade, da estratégia da empresa e dos resultados obtidos em $\mathrm{CL}_{1}$ e resultou na inclusão de dois novos requisitos no checklist. O primeiro requisito foi a capacidade técnica das empresas. Trata-se da comprovação de que os profissionais estão qualificados para exercer as atividades para as quais foram designados. Essa comprovação passa por uma descrição de cargos e funções, especificando os requisitos necessários para a atividade. $\mathrm{Na}$ análise da capacidade técnica se buscou verificar se os equipamentos de produção eram adequados para atendimento dos requisitos de qualidade demandados pelos produtos, como, por exemplo, a presença de máquinas de precisão para fabricação de peças com baixa tolerância dimensional. O segundo requisito adicionado foi a sustentabilidade, sendo que se avalia a segurança do trabalho e meio ambiente, as condições físicas de trabalho oferecidas pela empresa e o atendimento a legislação ambiental.

Um ano após a primeira aplicação do checklist nos fornecedores foi realizada a sétima etapa do procedimento metodológico, que consiste na reaplicação do checklist revisado e atualização das três parcelas do $I_{Q} F_{n, m}$. $O$ resultado final da reavaliação está apresentado na Tabela 3, comparando o resultado da reaplicação do checklist $\left(\mathrm{CL}_{2}\right)$ de cada requisito com a primeira avaliação $\left(\mathrm{CL}_{1}\right)$. Os novos 
requisitos adicionados ao checklist estão identificados pelas siglas R6 e R7 e tratam respectivamente da capacidade técnica e sustentabilidade.

Tabela 3 - Comparações de checklist e Índice de Qualificação de Fornecedor

\begin{tabular}{|c|c|c|c|c|c|c|c|c|c|c|c|c|c|c|c|c|}
\hline \multirow{2}{*}{ Forn. } & \multirow{2}{*}{$\mathrm{CL}_{1}$} & \multirow{2}{*}{$\mathrm{CL}_{2}$} & \multicolumn{2}{|c|}{ R1 } & \multicolumn{2}{|c|}{ R2 } & \multicolumn{2}{|c|}{ R3 } & \multicolumn{2}{|c|}{ R4 } & \multicolumn{2}{|c|}{ R5 } & \multirow{2}{*}{ R6 } & \multirow{2}{*}{ R7 } & \multirow{2}{*}{$I Q F_{1,1}$} & \multirow{2}{*}{ IQF $_{2,1}$} \\
\hline & & & $\mathrm{CL}_{1}$ & $\mathrm{CL}_{2}$ & $\mathrm{CL}_{1}$ & $\mathrm{CL}_{2}$ & $\mathrm{CL}_{1}$ & $\mathrm{CL}_{2}$ & $\mathrm{CL}_{1}$ & $\mathrm{CL}_{2}$ & $\mathrm{CL}_{1}$ & $\mathrm{CL}_{2}$ & & & & \\
\hline F1 & 63,33 & 85,70 & 3,33 & 3,00 & 2,00 & 2,00 & 2,67 & 3,33 & 1,50 & 3,75 & 4,00 & 4,00 & 4,00 & 4,00 & 80,03 & 82,86 \\
\hline $\mathrm{F} 2$ & 61,70 & 69,30 & 4,00 & 4,00 & 2,00 & 1,67 & 2,00 & 2,00 & 1,75 & 1,75 & 3,00 & 4,00 & 4,00 & 2,75 & 72,30 & 4,22 \\
\hline $\mathrm{F}^{*}{ }^{*}$ & 46,70 & 46,70 & 2,67 & 2,67 & 1,67 & 1,67 & 1,33 & 1,33 & 1,25 & 1,25 & 3,00 & 3,00 & NA & NA & 64,86 & 72,09 \\
\hline $\mathrm{F} 4$ & 80,00 & 89,70 & 3,67 & 3,67 & 3,00 & 3,67 & 3,00 & 3,67 & 3,25 & 3,25 & 4,00 & 4,00 & 3,33 & 3,00 & 80,68 & 83,12 \\
\hline F5 & 61,70 & 88,00 & 3,67 & 3,67 & 2,00 & 3,67 & 1,67 & 3,33 & 2,00 & 3,00 & 3,50 & 4,00 & 3,67 & 3,50 & 71,12 & 82,35 \\
\hline F6 & 70,00 & 84,00 & 3,33 & 3,67 & 2,00 & 3,33 & 1,33 & 3,00 & 3,50 & 3,00 & 4,00 & 3,00 & 3,33 & 3,25 & 74,81 & 81,06 \\
\hline F7 & 76,70 & 91,00 & 3,67 & 4,00 & 3,00 & 3,33 & 2,33 & 3,67 & 3,25 & 3,75 & 3,00 & 3,50 & 3,67 & 3,25 & 0 & 8,18 \\
\hline F8 & 50,00 & 66,20 & 2,67 & 1,33 & 2,00 & 2,67 & 1,00 & 3,67 & 1,50 & 2,75 & 3,50 & 3,00 & 3,00 & 2,50 & 47,47 & 72,48 \\
\hline $\mathrm{F}^{*}$ & 68,30 & 68,30 & 4,00 & 4,00 & 2,00 & 2,00 & 3,33 & 3,33 & 1,50 & 1,50 & 3,50 & 3,50 & NA & NA & 73,78 & 71,82 \\
\hline F10* & 90,00 & 90,00 & 3,67 & 3,67 & 4,00 & 4,00 & 2,67 & 2,67 & 3,75 & 3,75 & 4,00 & 4,00 & NA & NA & 85,77 & 93,67 \\
\hline F11 & 63,30 & 89,70 & 4,00 & 3,33 & 2,33 & 3,33 & 2,33 & 3,33 & 2,00 & 3,50 & 3,00 & 4,00 & 4,00 & 3,75 & 73,03 & 85,05 \\
\hline $\mathrm{F} 12^{*}$ & 71,70 & 71,70 & 4,00 & 4,00 & 1,67 & 1,67 & 3,00 & 3,00 & 2,25 & 2,25 & 4,00 & 4,00 & NA & NA & 83,45 & 80,06 \\
\hline Média & 66,95 & 78,36 & 3,56 & 3,42 & 2,31 & 2,75 & 2,22 & 3,03 & 2,29 & 2,79 & 3,54 & 3,67 & 3,63 & 3,25 & 74,63 & 80,58 \\
\hline
\end{tabular}

Observa-se que todos os fornecedores reavaliados aumentaram a sua pontuação $\mathrm{CL}_{n}$, indicando aderência ao plano de ação proposto pelos próprios fornecedores. Como informado anteriormente, dois fornecedores não executaram o plano de ação e sua reavaliação não foi realizada, além disso, outros dois fornecedores já homologados decidiram não realizar a segunda avaliação anual através do checklist, também alegando falta de tempo por suas direções. Estes fornecedores estão identificados pelo símbolo “*” e para fins de comparação suas notas da primeira avaliação foram replicadas para a segunda avaliação. Para as médias dos requisitos $\mathrm{R} 6$ e $\mathrm{R} 7$ estes fornecedores não foram considerados, tendo sua pontuação identificada como Não Avaliada (NA). A continuidade destas empresas no programa será analisada para futuras aplicações.

A análise dos dados mostrou que os requisitos que apresentaram maior evolução foram R3 e R4, com um aumento de $36 \%$ e $22 \%$ respectivamente em suas pontuações médias. O aumento observado no R3 deveu-se ao fato da verificação de elementos não identificados na avaliação anterior como, por exemplo, a folha de processo, indicando a sequência de atividades para o operador e o programa de 5S's estruturado na empresa fornecedora. O R4 apresentou evolução, mas ainda possui média de 2,79, considerada baixa. Nem todos os fornecedores adotaram um sistema de controle de calibração de instrumentos de medições, criaram e Revista Produção Online, Florianópolis, SC, v.13, n. 4, p. 1569-1595, out./dez. 2013. 
organizaram seus procedimentos escritos de qualidade, assim sendo estes dois fatores contribuíram negativamente para o desempenho de R4.

A média do R2 subiu 19 \% em relação à primeira avaliação dos fornecedores, atingindo 2,75 pontos. Este aumento foi impulsionado pela melhoria na rastreabilidade da matéria prima nas dependências dos fornecedores. A falta de um sistema de avaliação da cadeia de fornecimento das empresas ainda é um fator crítico para manutenção deste requisito com uma média considerada ruim pela empresa compradora. O R1 manteve-se nos mesmos patamares, apresentando uma variação de 3\% para menos, devido a um dos fornecedores ter regredido no seu método de rastreabilidade de orçamentos, reduzindo a média geral do grupo. Os dois novos requisitos (R6 e R7) tiveram médias de 3,63 e 3,25 respectivamente.

A partir da reaplicação do checklist, o IQF 2,1 foi atualizado com o índice $\mathrm{CL}_{2}$ gerado. A comparação entre $0 \operatorname{IQF}_{1,1}$ e $I_{2,1}$ está evidenciada na Tabela 3. Observa-se que os cinco fornecedores homologados no período anterior mantiveram-se homologados. Além deles, mais três fornecedores apresentaram pontuação acima de 80 , sendo considerados homologados também.

A oitava etapa ocorreu através de uma reunião entre a equipe do projeto para análise dos resultados ao longo do ano. Esta análise permitiu concluir que houve uma evolução na média do $\mathrm{IQF}_{n, m}$ dos fornecedores no período de um ano de programa, como mostra a Figura 5. Verificou-se que até $I Q F_{1,6}$ a média apresentavase crescente, a partir desta avaliação o $\mathrm{IQF}_{n, m}$ começou a apresentar uma redução devido a um mau desempenho nos indicadores $\mathrm{NC}_{n, m}$ ou $\mathrm{PE}{ }_{n, m}$. O resultado $\mathrm{IQF}_{2,1}$, que já possui nova parcela $\mathrm{CL}_{2}$, mostra que a média eleva-se de patamar, pois a maioria dos fornecedores apresentou evolução no índice $\mathrm{CL}_{2}$.

A análise individual das médias dos índices $\mathrm{NC}_{n, m}$ e $\mathrm{PE}_{n, m}$ confirma a análise feita sobre a média do $\mathrm{IQF}_{n, m}$ dos fornecedores. O resultado $\mathrm{NC}_{n, m}$ manteve-se crescente até a média $\mathrm{NC}_{1,6}$ quando sofreu uma redução, mantendo um índice $\mathrm{NC}_{n, m}$ médio de 85 pontos até $\mathrm{NC}_{1,12}$, quando novamente voltou a uma média de 86,6 pontos. De qualquer forma, observa-se uma mudança de patamar deste índice através da Figura 5. O índice $\mathrm{PE}_{n, m}$ é o que merece maior atenção, pois desde a implantação do programa, os fornecedores apresentaram uma redução na média deste índice, conforme mostra a Figura 5. 
A não evolução do indicador $\mathrm{PE}_{n, m}$ revela uma fragilidade no programa de avaliação. Esperava-se que uma evolução no índice $\mathrm{CL}_{n}$ revelasse um bom controle dos processos dos fornecedores que possibilitasse um bom desempenho tanto no índice $\mathrm{NC}_{n, m}$ quanto no $\mathrm{PE}_{n, m}$. Por outro lado, vale ressaltar que de acordo com a Equação 1 foi definido que $\mathrm{NC}_{n, m}$ teria um peso superior a $\mathrm{PE}_{n, m}$, o que pode ter sido um fator determinante para que os fornecedores definissem $\mathrm{NC}_{n, m}$ como prioritário em suas ações.

Concluiu-se também que a falta de um feedback estruturado com maior frequência para o fornecedor contribuiu para o não atingimento de um bom desempenho no índice $\mathrm{PE}_{n, m}$. O feedback a alta direção das empresas fornecedoras mostra-se como principal forma de manter as empresas cientes de suas limitações e comprometidas com a melhoria contínua, por isso se chegou a conclusão que tais ações devem ser realizadas no mínimo trimestralmente para futuras aplicações.

Figura 5 - Gráfico de evolução da média do IQF e dos índices PE e NC

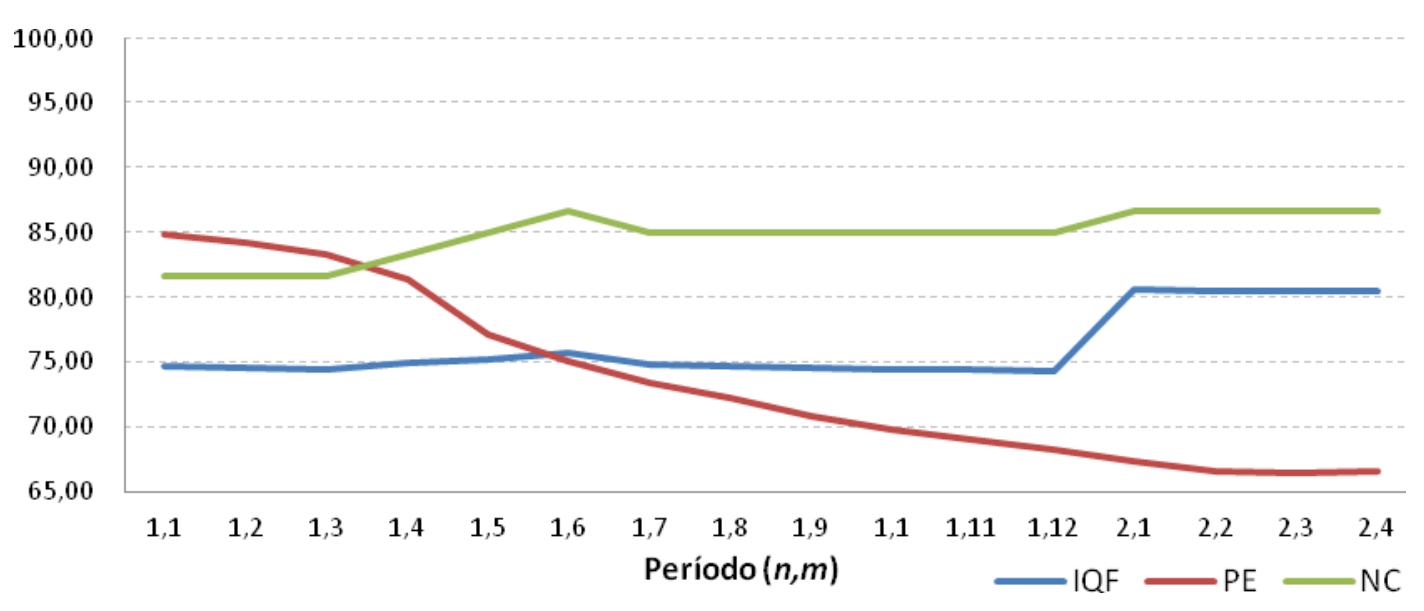

Concluiu-se que a aplicação de um ciclo completo do programa de avaliação atingiu resultados satisfatórios. Oito fornecedores completaram um ciclo completo de avaliações, dos quais seis foram considerados homologados. Devido a sua importância para empresa, foi decidido manter no programa os quatro fornecedores não reavaliados, que serão convidados a participar do novo ciclo de avaliações. Acredita-se que os resultados obtidos neste primeiro ciclo de avaliações poderão ser melhorados com a continuidade do programa, pois se espera que a valorização 
destas ações e o comprometimento dos fornecedores aumentem ao longo dos demais ciclos.

\section{CONCLUSÕES}

Este artigo apresentou a aplicação de um programa de avaliação de fornecedores de uma empresa siderúrgica multinacional, do qual participaram 12 fornecedores. O programa teve como objetivo monitorar e avaliar de maneira sistêmica as práticas e o desempenho de atendimento dos fornecedores através de um Índice de Qualificação de Fornecedores (IQF), calculado a partir da ponderação dos indicadores de pontualidade de entregas de materiais, de qualidade dos produtos fornecidos e da avaliação formal dos processos do fornecedor.

As etapas para desenvolvimento do programa foram conduzidas por uma equipe composta por integrantes dos setores de gestão da qualidade, suprimentos e administração de materiais da empresa estudada. Verificou-se que os fornecedores avaliados obtiveram uma evolução no seu IQF ao longo da execução do programa, impulsionado pela melhoria no desempenho dos índices de qualidade dos produtos e na avaliação formal dos processos das empresas através do checklist.

Uma redução do índice de não conformidades gerou ganhos para a empresa na redução do custo associado à interrupção da produção e no retrabalho das peças para os fornecedores. A evolução apresentada na avaliação através do checklist mostrou evidências de que os fornecedores estão tornando seus processos mais eficientes e como consequência tornando-se mais competitivos. Por outro lado, o retrocesso no desempenho do índice de pontualidade de entregas revela fragilidade no programa de avaliação. O peso dado a cada índice avaliado no IQF deve estar ajustado à estratégia da empresa para sua cadeia de fornecedores. No estudo de caso apresentado, optou-se em atribuir maior importância à qualidade dos materiais, o que pode ter acarretado em um desempenho insatisfatório no índice de pontualidade de entrega de materiais. Talvez um maior equilíbrio entre a importância atribuída a cada um dos índices refletisse em uma evolução no desempenho.

Efetuando as correções necessárias nas oportunidades de melhoria verificadas no programa de avaliação acredita-se que ele possa ser estendido a outros fornecedores da cadeia de suprimentos da empresa compradora ou a 
qualquer cadeia de fornecedores, desde que se realizem as adaptações necessárias. Verificou-se ainda que um sistema de avaliação cíclico, com fornecimento de feedback e comprovação de melhorias, mantêm os fornecedores em constante comprometimento com os resultados, contribuindo para uma cadeia mais competitiva.

\section{REFERÊNCIAS}

ALMEIDA, V.B. Programa de avaliação de fornecedores: um estudo de caso em uma empresa do setor siderúrgico. Trabalho de conclusão (graduação) - Escola de Engenharia, Curso de Engenharia de Produção, Universidade Federal do Rio Grande do Sul, Porto Alegre, 23 f, 2011.

ASSOCIAÇÃO BRASILEIRA DE NORMAS TÉCNICAS. NBR ISO 9001: sistemas de gestão da qualidade: requisitos. Rio de Janeiro, 2008.

ASSOCIAÇÃO BRASILEIRA DE NORMAS TÉCNICAS. Sistemas de gestão da qualidade: requisitos particulares para aplicação da ABNT NBR ISO 9001:2008 para organizações de produção automotiva e peças de reposição pertinentes. Rio de Janeiro, 2010.

BAILY, P.; DAVID, F.; DAVID, J. Compras princípios e administração. São Paulo: Atlas, 2000.

BALLOU, R.H. Gerenciamento da cadeia de suprimentos: planejamento, organização e logística empresarial. Porto Alegre: Bookman, 2001.

BERNARDI, A.C.; RODRIGUES, A.A.; MENDONÇA, F.C. Análise e melhoria do processo de avaliação dos impactos econômicos, sociais e ambientais de tecnologias da Embrapa Pecuária Sudeste. Gestão e Produção, v. 17, p. 297-316, 2010.

BIAZZO, S.; BERNARDI, G. Process management practices and quality systems standards. Business Process Management Journal, v. 9, n. 2., p. 149-169, 2003.

BIEHL, R.E. Customer-supplier analysis in education change. Quality Management Journal. v.7, n.2, p.22 - 39, 2000.

CAMPOS, V.F. Controle da qualidade total (no estilo japonês). Belo Horizonte: Fundação Christiano Ottoni, 1992.

CAMPOS, V.F. Gerenciamento da rotina do trabalho do dia-a-dia. Belo Horizonte: Editora de Desenvolvimento Gerencial, 1998.

CARTER, J.R.; SMELTZER L.; NARASIMHAN, R. The role of buyer and supplier relationships in integrating tqm through the supply chain. European Journal of Purchasing \& Supply Management, v.4, n.4, p.223 - 234, 1998. 
CRAWFORD, K.M.; COX, J.F. Addressing manufacturing problems through the implementation of Just-in-time. Production and Inventory Management Journal, v.32, n.1, p.33 - 36, 1991.

DEUS, A.; VACCARO, G.L.R. Uma abordagem para implementação de qualidade assegurada no fornecimento, baseada em análise de capacidade: um estudo de caso em uma empresa do setor automotivo. Revista Produção Online, v. 9, n.4, 2009.

ESTORILIO, C.; POSSO, R. Redução das irregularidades identificadas na aplicação do fmea de processo: um estudo em produtos estampados. Revista Produção Online, v.11, n.4, 2011.

FILHO, C.; JUNIOR, L.C.; COSTA, M.G. Indústria de cartucho de toner sob a ótica da remanufatura: estudo de caso de um processo de melhoria. Revista Produção, v.16, p. 100-110, 2006.

FU, Y.; PIPLANI, R. Supply-side collaboration and its value in supply chains. European Journal of Operational Research, v. 152, p. 281-288, 2004.

GIL, A.C. Como elaborar projetos de pesquisa. São Paulo: Atlas, 2010.

GRIFFITHS, J.; MARGETTS D. Variation in production schedules - implications for both the company and its suppliers. Journal of Materials Processing Technology, v.103, n.1, p.155 - 159, 2000.

HARO, D.G. Sistemas da qualidade na indústria automobilística: uma proposta de auto-avaliação unificada. Dissertação (Mestrado Profissionalizante em Engenharia). Escola de Engenharia, Programa de Pós-Graduação em Engenharia de Produção, Universidade Federal do Rio Grande do Sul, Porto Alegre, 136 f., 2001.

HUMPHREYS, P.K.; SHIU, W.K.; CHAN, F.T.S. Collaborative buyer-supplier relationships in Hong Kong manufacturing firms. Supply Chain Management: An International Journal, v.6, n.4, p. 152 - 162, 2001.

ISHIKAWA, K. Controle da qualidade total: a maneira japonesa. Rio de Janeiro: Campus, 1993.

KARTA, C.P. A comparison of ISO 9000:2000 quality system standards, QS9000, ISO/TS 16949 and Baldrige criteria. The TQM Magazine, v.16, p. 331,340, 2004.

KRALJIC, P. Purchasing must become supply management. Harvard Business Review, v. 61, p. 109-117, 1983.

KRAUSE, D. Supplier development: current practices and outcomes. International Journal of Purchasing and Material Management, v. 33, p 12-19, 1997.

KRAUSE, D. The antecedents of buying firm's efforts to improve suppliers. Journal of Operations Management, v.17, n.2, p.205 - 224, 1999. 
KRAUSE, D.; ELLRAM, L.M. Critical elements of supplier development. European Journal of Purchasing and Supply Management, v. 3, p. 21- 31, 1997.

LAMBERT, D.M.; COOPER, M.C. Issues in supply chain management. The International Journal of Logistics Management, v. 29, p. 65-83, 2000.

LAMMING, R. Beyond partnership: strategies for innovation and lean supply. Hemmel Hempstead: Prentice-Hall, 1993.

MELLO, C.H.P.; SILVA, C.E.S.; TURRIONI, J.B.; SOUZA, L.G.M. ISO 9001:2008: sistema de gestão da qualidade para operações de produção e serviços. São Paulo: Atlas, 2009.

MERLI, G. Comarkership: a nova estratégia para os suprimentos. Rio de Janeiro: Qualytmark, 1998.

MILGATE, M. Supply chain complexity and delivery performance: an international exploratory study. Supply Chain Mangement: an International Journal, v.6, n.3, p. $106-118,2001$.

MOHR, J.; SPEKMAN, R. Characteristics of partnership success: partnership attributes, communication behavior, and conflict resolution techniques. Strategic Management Journal, v.15, n.2, p.135 - 152, 1994.

NEUMANN, C.S.R.; RIBEIRO, J.L. Desenvolvimento de fornecedores: um estudo de caso utilizando a troca rápida de ferramentas. Revista Produção, v. 14, p. 44-53, 2004.

ROSA, E. P.; SELLITO, M.A.; MENDES, L.W. Avaliação multicriterial de desempenho e separação em aglomerados de fornecedores críticos de uma manufatura OKP. Revista Produção, v. 16, p. 413-428, 2006.

SEIDEL, E.J.; LOPES, L.F.D.; ANSUJ, A.P.; ZANELLA, A. Métodos estatísticos aplicados à avaliação da qualidade da matéria-prima e classificação dos fornecedores de uma indústria de laticínios. Revista Produção Online, v.11, n.1, 2011.

SILVA, E.L.S.; MENEZES, E.M. Metodologia de pesquisa e elaboração de dissertação. Florianópolis: UFSC, 2005.

STUART, F.I.; McCUTCHEON, D. Sustaining strategic supplier alliances - profiling the dynamic requeriments for continued development. International Journal of Operations \& Production Management, v.16, n.10, p.5 - 22, 1996.

STUELAND, V.J. Supplier evaluation: best practices and creating or improving your own evaluation. In International Supply Management Conference, 89, 2004, Philadelphia. International Conference Proceedings. Tempe: Institute for Supply Management, 2004. Disponível em http://www.ism.ws/pubs/proceedings >. Acesso em: 20 jan. 2011. 
TAYLOR, D.; BRUNT, D. Manufacturing operations and supply chain management: the lean approach. London: Thomson Learning, 2001.

TRACEY, M.; TAN, C.L. Empirical analysis of supplier selection and involvement, customer satisfaction, and firm performance. Supply Chain Management, v. 6, p. 174-188, 2001.

TUKEL, O.I.; WASTI, S.N. Analysis of supplier buyer relationships using resource constrained project scheduling strategies. European Journal of Operational Research, v.129, n.2, p.271-276, 2001.

WATTS, C.A.; HAHN, C.K. Supplier development programs: an empirical analysis. International Journal of Purchasing and Materials Management, v.29, n.2, p.10 $17,1993$.

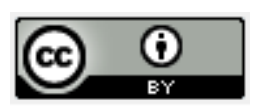

Artigo recebido em 26/02/2013 e aceito para publicação em 20/06/2013. 